Proceedings

\title{
Determination of Intrinsic and Metrological Correlations of Components of Product Impact on Risks of False Decisions in Conformity Assessment ${ }^{\dagger}$
}

\author{
Luciana Separovic ${ }^{1, *}$, Ricardo J. N. Bettencourt da Silva ${ }^{2}$ and Felipe Rebello Lourenço ${ }^{1}$ \\ 1 Departamento de Farmácia, Faculdade de Ciências Farmacêuticas, Universidade de São Paulo, \\ 05508-000 São Paulo, Brazil; feliperl@usp.br \\ 2 Centro de Química Estrutural, Faculdade de Ciências da Universidade de Lisboa, \\ 1749-016 Lisbon, Portugal; rjsilva@fc.ul.pt \\ * Correspondence: luseparovic@usp.br \\ † Presented at the Virtual Eurachem Workshop 2020 - "Quality Assurance for Analytical Laboratories in the \\ University Curriculum", 14-15 July 2020; Available online: https;//eurachem2020.ro/.
}

Published: 18 September 2020

\begin{abstract}
The correlation between the measured values of product components can influence the total risk of false decisions in conformity assessment. This correlation can originate from the characteristics of the product (intrinsic) or from how the components are measured (metrological). This work aimed to determine both correlations by testing a medicine with two compounds separately and together (correlated). The same intrinsic correlation was estimated regardless of whether the measurements were independent or correlated. Furthermore, the intrinsic covariance contributed significantly to the total covariance between the components evaluated. Both metrological and intrinsic correlations should be considered, as they can affect the risks of false decisions in conformity assessment when there are two or more compounds associated in the product.
\end{abstract}

Keywords: covariance; correlation; drug association; conformity assessment

\section{Introduction}

Conformity assessment of a product or item may require the evaluation of multiple quality parameters. To make this assessment reliable, the measurement uncertainty associated with each measured value must be taken into account when comparing them with the specification limits. When measurement uncertainty is neglected, the risks of false decisions in conformity assessment are unknown, therefore, they cannot be subject to strategies to minimize them, and the rate of false product acceptance or rejection can be inadequately high [1].

One factor that can influence the total risk value (which takes into account all quality parameters of a product) is the correlation between the measured values of the product components. The correlation between these values can originate from the characteristics of the product acquired from its production, as in medicines that have active compounds associated (material or intrinsic correlation), or from how the components are measured (artificial or metrological correlation) $[2,3]$. Given the above, this work aimed to determine how the experimentally observed correlation between measured values of a product's components (total correlation) can be decomposed into the intrinsic and metrological correlations, and how they can affect the total risk of a false decision in conformity assessment. 


\section{Materials and Methods}

In this work, the assay of amiloride hydrochloride $5 \mathrm{mg}$ + hydrochlorothiazide $50 \mathrm{mg}$ tablets (12 batches), described in the Brazilian Pharmacopeia [4], was studied.

First, the batches' content of hydrochlorothiazide and amiloride hydrochloride were estimated separately, with independent samples and standards preparation for the assay of each compound. Then, the assay was performed considering the contents of both compounds together, that is, with shared analytical steps which leads to metrological correlation.

\section{Results and Discussion}

The following were calculated from the measurement results: the total variance of each measured value of the product components and the total covariance and total correlation between them for the independent and correlated measurements (Table 1).

The expanded measurement uncertainty (U) was determined by combining the uncertainty components using the Kragten method, and the following values were determined: $U=1.94 \%$ (percentage of the product specification as analyte mass per tablet) for hydrochlorothiazide (HCT) and $\mathrm{U}=2.10 \%$ for amiloride hydrochloride (AMIL; coverage factor of 2 for approximately 95\% confidence level). Furthermore, from the correlation matrix built using a previously developed tool [2], it was possible to obtain the values of the metrological variance and covariance (Table 1).

The intrinsic covariance can be estimated by the difference between the total covariance and the metrological covariance, considering the property of linear combinations of covariances $[5,6]$. Considering the metrological component, only the components that contribute to the experimentally determined total variance should be considered:

$$
\operatorname{cov}(X+Y, W+Z)=\operatorname{cov}(X, W)+\operatorname{cov}(X, Z)+\operatorname{cov}(Y, W)+\operatorname{cov}(Y, Z)
$$

where $\mathrm{X}+\mathrm{Y}$ and $\mathrm{W}+\mathrm{Z}$ represent the linear combination of variables representing the intrinsic $(\mathrm{X}$ or $\mathrm{W})$ and metrological $(\mathrm{Y}$ or $\mathrm{Z})$ variance components associated with the first $(\mathrm{X}+\mathrm{Y})$ and second $(\mathrm{W}+$ Z) determined chemical parameters of the product (in this work, HCT and AMIL).

As the value due to the production process of one component is independent from the measurement of the other component (i.e., $\operatorname{cov}(X, Z)=0$ and $\operatorname{cov}(Y, W)=0$ ), then:

$$
\operatorname{cov}(X+Y, W+Z)=\operatorname{cov}(X, W)+\operatorname{cov}(Y, Z),
$$

where $\operatorname{cov}(X+Y, W+Z)$ is the total/experimental covariance, $\operatorname{cov}(X, W)$ is the intrinsic covariance, and $\operatorname{cov}(\mathrm{Y}, \mathrm{Z})$ is the metrological covariance.

Thus, the intrinsic variances to the product components were obtained from the difference between the total and metrological variances for each component, and the intrinsic covariance was obtained by the difference between the total and metrological covariances. Table 1 presents the calculated values of variance, correlation and covariance components from independent and correlated determinations of both components.

Table 1. Total, metrological and intrinsic variances (Var), covariance (Cov), and correlation for hydrochlorothiazide (HCT) and amiloride hydrochloride (AMIL) measured independently or correlated.

\begin{tabular}{ccccccc}
\hline & \multicolumn{3}{c}{ Independent Measurements } & \multicolumn{3}{c}{ Correlated Measurements } \\
\hline & Total & Metrological & Intrinsic & Total & Metrological & Intrinsic \\
\hline Cov $\left(\%^{2}\right)$ & $8.006-$ & $0=$ & 8.006 & $7.463-$ & $0.9931=$ & 6.470 \\
Var HCT $\left(\%{ }^{2}\right)$ & $6.110-$ & $0.9488=$ & 5.161 & $6.800-$ & $0.9488=$ & 5.851 \\
Var AMIL $\left(\%^{2}\right)$ & $17.61-$ & $1.094=$ & 16.51 & $11.09-$ & $1.094=$ & 9.999 \\
Correlation & $0.7719-$ & $0=$ & 0.8672 & $0.8593-$ & $0.9746=$ & 0.8459 \\
\hline
\end{tabular}

It was observed from the results of correlated measurements that $86.7 \%(6.470 / 7.463)$ of the total covariance came from the intrinsic covariance of the product components (Table 1), which can be 
explained by the low measurement uncertainties (metrological HCT and AMIL Var of 0.949 and 1.09 , respectively).

The intrinsic correlations were similar considering independent and correlated measurements ( 0.8672 and 0.8459 , respectively $-p$-value 0.184 via Monte Carlo simulations). Therefore, we were able to conclude that the same intrinsic correlation was estimated regardless of whether the measurements were independent or correlated, proving the adequacy of the considered algorithms and uncertainty evaluation approach. On the other hand, we found significant statistical differences between the total correlations obtained from independent and correlated measurements $(0.7719$ and 0.8593 , respectively $-p$-value 0.004 via Monte Carlo simulations). Thus, we conclude that metrological correlation due to sharing relevant analytical steps may significantly affect total correlation.

\section{Conclusions}

This work demonstrates that the intrinsic covariance of pairs of product components can be calculated from the difference between the total covariance experimentally observed and the metrological covariance. Moreover, the intrinsic covariance contributed significantly to the total covariance between the components evaluated. However, if measured values present higher uncertainty, the metrological correlation may be more significant. Therefore, it is important to consider to what extent the metrological and intrinsic correlations influence the total correlation, as they consequently also affect the risks of false decisions in conformity assessment when there are two or more compounds associated in the product.

Whenever the metrological correlation affects conformity decisions significantly, the measurement process should be reviewed to reduce this impact. The management of intrinsic correlations is also possible.

Funding: This research was funded by FAPESP - Fundação de Amparo à Pesquisa do Estado de São Paulo (Brazil), grant number 2017/04539-2 and 2018/25734-0.

Conflicts of Interest: The authors declare no conflict of interest, and the funders had no role in the design of the study; in the collection, analyses, or interpretation of data; in the writing of the manuscript; or in the decision to publish the results.

\section{References}

1. Luciana, S.; Felipe, R.L. Measurement uncertainty and risk of false conformity decision in the performance evaluation of liquid chromatography analytical procedures. J. Pharm. Biomed. Anal. 2019, 171, 73-80.

2. Luciana, S.; Ricardo, J.N.B.S.; Felipe, R.L. Improved spreadsheet method for determination of between components metrological correlation due to the sharing of analytical steps. Chemom. Intell. Lab. Syst. 2019, 189, 161-168.

3. Felipe, R.L.; Ricardo, J.N.B.S. Risk of false conformity decisions of multicomponent items controlled by correlated measurement results due to the sharing of analytical steps. Talanta 2019, 196, 174-181.

4. Agência Nacional de Vigilância. Sanitária Farmacopeia Brasileira; Agência Nacional de Vigilância: Brasília, Brazil, 2010; Volume 2, pp. 805-806.

5. Randall, J.P. Foundations and Applications of Statistics: An Introduction Using R, 1st ed.; Amer Mathematical Society: Providence, RI, USA, 2011; p. 615.

6. Michael, W.W.; Whitley, Jr.; Bernard, E. (Eds.) Handbook for Conducting Research on Human Sexuality, 1st ed.; Lawrence Erlbaum Associates Inc.: Mahwah, NJ, USA, 2002; p. 544. 